\title{
Unknown Input Sliding Mode Functional Observers with Application to Sensorless Control of Permanent Magnet Synchronous Machines
}

\author{
Sanath Alahakoon ${ }^{\mathrm{a}}$, Tyrone Fernando ${ }^{\mathrm{b}, *}$, Hieu Trinh ${ }^{\mathrm{c}}$, Victor Sreeram ${ }^{\mathrm{b}}$ \\ ${ }^{a}$ Central Queensland University, Brian Jordan Drive, Gladstone, Queensland 4680, \\ Australia. \\ ${ }^{b}$ School of Electrical, Electronic and Computer Engineering, University of Western \\ Australia, 35 Stirling Hwy, Crawley, WA 6009, Australia. \\ ${ }^{c}$ School of Engineering, Deakin University, Geelong, VIC 3217, Australia.
}

\begin{abstract}
This paper illustrates a method of designing a sliding mode linear functional observer for a system with unknown inputs. The existence conditions for the observer are presented. A structure and design algorithm for the sliding mode observer is proposed. The proposed algorithm is then applied for sensorless control of Permanent Magnet Synchronous Machines.
\end{abstract}

Keywords:

Functional Observability, Functional Detectability, Functional Observers, Minimum Order Observers

\section{Introduction}

Sliding mode observers differ from more traditional observers e.g. Luenberger observers, in that there is a non-linear term injected into the observer depending on the output estimation error. The concept of sliding mode was originally applied to control system design [1], [2] and later applied for estimating system states [2],[3],[4]. State estimators that utilize the concept

\footnotetext{
${ }^{*}$ Corresponding author

Email addresses: s.alahakoon@cqu.edu.au (Sanath Alahakoon), tyrone@ee.uwa.edu.au (Tyrone Fernando), Hieu.Trinh@deakin.edu.au (Hieu Trinh), sreeram@ee.uwa.edu.au (Victor Sreeram)
} 
of sliding mode are referred to as Utkin observers in the literature [3]. Sliding mode functional observers estimate linear functions of the state vector of a system without necessarily estimating all of the individual states while ensuring that sliding will occur on a manifold where some function of the output prediction error is zero. Since only required functions are estimated with functional observers, these observers have lower order than full order observers, the order of the functional observer can be as low as the number of functions estimated. Much of the work here has been motivated by the work of [5] and [6], where the importance of sliding modes is demonstrated in Variable Structure Systems. The practical applications of sliding mode observers in power and control have been illustrated in [7],[8],[9] and [10] most importantly in the field of induction motor control. It is also noted in [11] and also in other articles on sliding mode observers [12], [13] the robustness properties of these type of observers. Motivated by reported robustness properties of sliding mode observers, in this paper we present the concept of sliding mode functional observers and apply it for sensorless control of Permanent Magnet Synchronous Motors (PMSM).

One of the most intriguing aspects of sliding modes as described in [3] is to switch between two distinctively different system structures (or components) such that a new type of system motion, sliding mode, exists in a manifold. This certain peculiar system characteristic which is a consequence of the switching function is claimed to result in superb system performance which includes insensitivity to parameter variations, and complete rejection of disturbances [3]. These properties of sliding mode are investigated in this paper by simulating the proposed observer in sensorless control of PMSMs.

In literature the state estimation problem for unknown input systems is well researched [14], [15], [16] however application of sliding mode concepts to estimate a linear function of the state vector has not yet been addressed. In this paper, we present conditions for designing sliding mode functional observers when the system is subjected to unknown inputs. Under special circumstances, the existence conditions derived in this paper reduces to the existence conditions for the Utkin Observer and also reduces to the well known matching and observability conditions required for the design of an unknown input state observer.

This paper is organized as follows: Section II provides a general outline of the problem to be solved. This section will provide a description to the unknown input system of equations and the corresponding sliding mode linear functional observer structure that will be applied. Section III describes the 
conditions for the existence of the sliding mode functional observer and based on these conditions a design algorithm is presented. Section IV provides the application of the theory to speed sensorless control of PMSMs. Finally, Section V presents the conclusions of the paper.

\section{Problem Statement}

Consider a linear time-invariant system described by

$$
\begin{aligned}
\dot{x}(t) & =A x(t)+B u(t)+D v(t) \\
y(t) & =C x(t) \\
z(t) & =L x(t)
\end{aligned}
$$

where $x(t) \in \mathbb{R}^{n}, u(t) \in \mathbb{R}^{m}, v(t) \in \mathbb{R}^{q}$ and $y(t) \in \mathbb{R}^{p}$ are the state, known input, unknown input and the output vectors, respectively. $z(t) \in \mathbb{R}^{r}$ is the vector to be estimated. The pair $(C, A)$ is detectable, $(A, B)$ is controllable, $A \in \mathbb{R}^{n \times n}, B \in \mathbb{R}^{n \times m}, C \in \mathbb{R}^{p \times n}, D \in \mathbb{R}^{n \times q}$ and $L \in \mathbb{R}^{r \times n}$ are known constant matrices. It is noted here that $D$ represents the unknown input matrix. Without loss of generality, it is assumed that $\operatorname{Rank}(C)=p, \operatorname{Rank}(L)=r$,

$\operatorname{Rank}\left[\begin{array}{l}L \\ C\end{array}\right]=r+p-\tilde{r}, \tilde{r} \leq r$ and $C$ takes the form $C=\left[\begin{array}{ll}I_{p} & 0\end{array}\right]$ (otherwise the system can always be transformed into this form).

Consider the following sliding mode linear functional observer

$$
\begin{aligned}
\dot{w}(t) & =N w(t)+J y(t)+H u(t)+\Gamma \operatorname{sgn}(M e(t)) \\
\hat{z}(t) & =w(t)+E y(t) \\
e(t) & =z(t)-\hat{z}(t)
\end{aligned}
$$

where $w(t) \in \mathbb{R}^{r}, M \in \mathbb{R}^{\tilde{r} \times r}, \Gamma \in \mathbb{R}^{r \times \tilde{r}}, \operatorname{sgn}(\cdot)$ is the sign function and also the sliding surface is given by

$$
M e(t)=0
$$

In view of the dimension of matrix $M$, the error vector $e(t)$ can be written as

$$
e(t)=\left[\begin{array}{l}
e_{y}(t) \\
e_{1}(t)
\end{array}\right]
$$

where $e_{y}(t) \in \mathbb{R}^{\tilde{r}}$ and $e_{1}(t) \in \mathbb{R}^{r-\tilde{r}}$.

The aim of this paper is to design a sliding mode functional observer, that is to find $N, J, H, E$ and a suitable $\Gamma$ such that $e(t)$ slides along the surface $M e(t)=0$ and $e_{1}(t) \rightarrow 0$ as $t \rightarrow \infty$. 


\section{Existence Conditions of the Observer}

To define the sliding surface the matrix $L$ can be partitioned as

$$
L=\left[\begin{array}{l}
L_{1} \\
L_{2}
\end{array}\right]
$$

where $L_{1}$ belong to the rowspace of $C$. Since $L_{1}$ belong to the rowspace of $C$, there always exist a full row rank matrix $G \in \mathbb{R}^{\tilde{r} \times p}, \tilde{r} \leq r$ such that

$$
L_{1}=G C
$$

If $M \in \mathbb{R}^{\tilde{r} \times r}$ is chosen such that

$$
M=\left[\begin{array}{ll}
I_{\tilde{r}} & 0_{\tilde{r} \times(r-\tilde{r})}
\end{array}\right]
$$

and $G$ according to (10), then the sliding surface (7) is

$$
M e(t)=e_{y}(t)=G y(t)-M \hat{z}(t)=0
$$

Remark 1: It is noted if $L$ and $C$ are linearly independent then $\tilde{r}=0$ and then according to (12) there is no sliding surface in the observer error space. If $\tilde{r}=0$ then any linear combinations of $C$ can be included in $L$ to define a sliding surface according to (12).

Using (1)-(6) and (12) the error dynamics of the observer can be written as follows

$$
\begin{array}{r}
\dot{e}(t)=N e(t)+(P A-N P-J C) x(t)+(P B-H) u(t) \\
+P D v(t)-\Gamma \operatorname{sgn}\left(e_{y}(t)\right)
\end{array}
$$

where $P=L-E C$. Considering the partitioning of $e(t)$ in (8), matrix $N$ can be partitioned as follows

$$
N=\left[\begin{array}{ll}
N_{11} & N_{12} \\
N_{21} & N_{22}
\end{array}\right]
$$

where, $N_{11} \in \mathbb{R}^{\tilde{r} \times \tilde{r}}, N_{12} \in \mathbb{R}^{\tilde{r} \times(r-\tilde{r})}, N_{21} \in \mathbb{R}^{(r-\tilde{r}) \times \tilde{r}}$ and $N_{22} \in \mathbb{R}^{(r-\tilde{r}) \times(r-\tilde{r})}$. If we now choose $\Gamma=\left[\begin{array}{l}\Gamma_{1} \\ 0_{(r-\tilde{r}) \times \tilde{r}}\end{array}\right], \Gamma_{1} \in \mathbb{R}^{\tilde{r} \times \tilde{r}}$ then the error dynamics (13) can be rewritten as

$$
\begin{aligned}
{\left[\begin{array}{c}
\dot{e}_{y}(t) \\
\dot{e}_{1}(t)
\end{array}\right]=} & {\left[\begin{array}{ll}
N_{11} & N_{12} \\
N_{21} & N_{22}
\end{array}\right]\left[\begin{array}{c}
e_{y}(t) \\
e_{1}(t)
\end{array}\right] } \\
& +(P A-N P-J C) x(t)+(P B-H) u(t) \\
& +P D v(t)-\left[\begin{array}{c}
\Gamma_{1} \operatorname{sgn}\left(e_{y}(t)\right) \\
0
\end{array}\right]
\end{aligned}
$$


The existence conditions of the observer (4)-(6) are given in the following Theorem.

Theorem 1: $e_{1}(t) \rightarrow 0$ as $t \rightarrow \infty$ and also $e(t)$ slide along the surface $M e(t)=0, t \geq t_{s}$ where $t_{s} \leq\left(\frac{\|G y(0)-M \hat{z}(0)\|}{\eta}\right), \eta>0$ for any $x(0), \hat{z}(0)$, and $u(t)$ if the following conditions hold:

$$
\begin{aligned}
& N_{22}, \text { Hurwitz } \\
& P A-N P-J C=0 \\
& H=P B \\
& P D=0 \\
& e_{y}^{T}(t) \dot{e}_{y}(t)<-\eta\left\|e_{y}(t)\right\|
\end{aligned}
$$

where, \|. $\|$ represents the norm

Proof: If Conditions (17) and (18) and (19) are satisfied then the error dynamics (15) of the observer is given by

$$
\begin{aligned}
& \dot{e}_{y}(t)=N_{11} e_{y}(t)+N_{12} e_{1}(t)-\Gamma_{1} \operatorname{sgn}\left(e_{y}(t)\right) \\
& \dot{e}_{1}(t)=N_{21} e_{y}(t)+N_{22} e_{1}(t)
\end{aligned}
$$

If (20) is satisfied then an ideal sliding motion will take place on the surface

$$
S_{0}=\left\{\left(e_{1}(t), e_{y}(t)\right): e_{y}(t)=0\right\}
$$

It follows that after some finite time $t_{s}$, for all subsequent time, $e_{y}=0[2]$. The dynamics of $e_{1}(t)$ then reduces to

$$
\dot{e}_{1}(t)=N_{22} e_{1}(t)
$$

If (16) is satisfied then $e_{1}(t) \rightarrow 0$ as $t \rightarrow \infty$.

Considering the partitioning of $e(t)$ in (8), matrices $P, J, E$ and $H$ can be partitioned as $P=\left[\begin{array}{l}P_{1} \\ P_{2}\end{array}\right], J=\left[\begin{array}{l}J_{1} \\ J_{2}\end{array}\right], E=\left[\begin{array}{c}E_{1} \\ E_{2}\end{array}\right]$ and $H=\left[\begin{array}{c}H_{1} \\ H_{2}\end{array}\right]$ where $P_{1} \in \mathbb{R}^{\tilde{r} \times n}, P_{2} \in \mathbb{R}^{(r-\tilde{r}) \times n}, J_{1} \in \mathbb{R}^{\tilde{r} \times p}, J_{2} \in \mathbb{R}^{(r-\tilde{r}) \times p}, E_{1} \in \mathbb{R}^{\tilde{r} \times p}$, $E_{2} \in \mathbb{R}^{(r-\tilde{r}) \times p}, H_{1} \in \mathbb{R}^{\tilde{r} \times m}$ and $H_{2} \in \mathbb{R}^{(r-\tilde{r}) \times m}$. Using the definition of $P=L-E C$, the partitioned matrices $P_{1}$ and $P_{2}$ can be written as

$$
\begin{aligned}
& P_{1}=L_{1}-E_{1} C \\
& P_{2}=L_{2}-E_{2} C
\end{aligned}
$$


and using (18) $H_{1}$ and $H_{2}$ can be written as

$$
\begin{aligned}
& H_{1}=P_{1} B \\
& H_{2}=P_{2} B
\end{aligned}
$$

Furthermore, equation (17) can be rewritten as

$$
\begin{aligned}
& P_{1} A-N_{11} P_{1}-N_{12} P_{2}-J_{1} C=0 \\
& P_{2} A-N_{21} P_{1}-N_{22} P_{2}-J_{2} C=0
\end{aligned}
$$

Equation (29) is equivalent to

$$
\left(P_{1} A-N_{11} P_{1}-N_{12} P_{2}-J_{1} C\right)\left[\begin{array}{ll}
L^{+} & I-L^{+} L
\end{array}\right]=0
$$

because $\left[L^{+} I-L^{+} L\right]$ is a full row rank matrix. Let us now partition $L^{+}$ as follows

$$
L^{+}=\left[\begin{array}{ll}
M_{1} & M_{2}
\end{array}\right]
$$

where $M_{1} \in \mathbb{R}^{n \times \tilde{r}}, M_{2} \in \mathbb{R}^{n \times(r-\tilde{r})}$ and [.] ${ }^{+}$represents the pseudoinverse. Using (25) and (32) and also the fact that $L_{1} M_{1}=I_{\tilde{r}}, L_{1} M_{2}=0, L_{2} M_{1}=0$, $L_{2} M_{2}=I_{(r-\tilde{r})}$, equation (31) leads to

$$
\begin{aligned}
& N_{11}=\left[L_{1} A M_{1}\right]-\left[\begin{array}{ll}
E_{1} & K_{1}
\end{array}\right]\left[\begin{array}{c}
C A M_{1} \\
C M_{1}
\end{array}\right] \\
& N_{12}=\left[L_{1} A M_{2}\right]-\left[\begin{array}{ll}
E_{1} & K_{1}
\end{array}\right]\left[\begin{array}{c}
C A M_{2} \\
C M_{2}
\end{array}\right] \\
& {\left[\begin{array}{ll}
E_{1} & K_{1}
\end{array}\right] \Sigma=\left[\begin{array}{ll}
L_{1} \bar{A} & L_{1} D
\end{array}\right]}
\end{aligned}
$$

where

$$
\begin{aligned}
& K_{1}=J_{1}-N_{11} E_{1}-N_{12} E_{2} \\
& \Sigma=\left[\begin{array}{cc}
C \bar{A} & C D \\
\bar{C} & 0
\end{array}\right] \\
& \bar{A}=A\left(I-L^{+} L\right) \\
& \bar{C}=C\left(I-L^{+} L\right)
\end{aligned}
$$

It can be shown that (35) can always be solved for $E_{1}$ and $K_{1}$ because $\left[\begin{array}{cc}L_{1} \bar{A} & L_{1} D\end{array}\right]\left(I-\Sigma^{+} \Sigma\right)=0$ (i.e. Rank $\left.\left[\begin{array}{cc}L_{1} \bar{A} & L_{1} D \\ \Sigma\end{array}\right]=\operatorname{Rank}[\Sigma]\right)$ and the general solution is given by

$$
\left[\begin{array}{ll}
E_{1} & K_{1}
\end{array}\right]=\left[\begin{array}{ll}
L_{1} \bar{A} & L_{1} D
\end{array}\right] \Sigma^{+}+Z_{1}\left(I-\Sigma \Sigma^{+}\right)
$$


where $Z_{1}$ is any arbitrary matrix of appropriate dimension. Using (40) in (33) and (34), the following can be written

$$
\begin{aligned}
& N_{11}=F_{11}-Z_{1} G_{1} \\
& N_{12}=F_{12}-Z_{1} G_{2}
\end{aligned}
$$

where

$$
\begin{aligned}
F_{11} & =\left[L_{1} A M_{1}\right]-\left[\begin{array}{ll}
L_{1} \bar{A} & L_{1} D
\end{array}\right] \Sigma^{+}\left[\begin{array}{c}
C A M_{1} \\
C M_{1}
\end{array}\right] \\
F_{12} & =\left[L_{1} A M_{2}\right]-\left[\begin{array}{ll}
L_{1} \bar{A} & L_{1} D
\end{array}\right] \Sigma^{+}\left[\begin{array}{c}
C A M_{2} \\
C M_{2}
\end{array}\right] \\
G_{1} & =\left(I-\Sigma \Sigma^{+}\right)\left[\begin{array}{c}
C A M_{1} \\
C M_{1}
\end{array}\right] \\
G_{2} & =\left(I-\Sigma \Sigma^{+}\right)\left[\begin{array}{c}
C A M_{2} \\
C M_{2}
\end{array}\right]
\end{aligned}
$$

As with (29), we can adopt the same procedure starting from equation (30). Equation (30) is equivalent to

$$
\left(P_{2} A-N_{21} P_{1}-N_{22} P_{2}-J_{2} C\right)\left[L^{+} I-L^{+} L\right]=0
$$

because $\left[L^{+} \quad I-L^{+} L\right]$ is a full row rank matrix. Using (26) and (32) and also the fact that $L_{1} M_{1}=I_{\tilde{r}}, L_{1} M_{2}=0, L_{2} M_{1}=0, L_{2} M_{2}=I_{(r-\tilde{r})}$, equation (47) leads to

$$
\begin{aligned}
& N_{21}=\left[L_{2} A M_{1}\right]-\left[\begin{array}{ll}
E_{2} & K_{2}
\end{array}\right]\left[\begin{array}{c}
C A M_{1} \\
C M_{1}
\end{array}\right] \\
& N_{22}=\left[L_{2} A M_{2}\right]-\left[\begin{array}{ll}
E_{2} & K_{2}
\end{array}\right]\left[\begin{array}{c}
C A M_{2} \\
C M_{2}
\end{array}\right] \\
& {\left[\begin{array}{ll}
E_{2} & K_{2}
\end{array}\right] \Sigma=\left[\begin{array}{ll}
L_{2} \bar{A} & L_{2} D
\end{array}\right]}
\end{aligned}
$$

where

$$
K_{2}=J_{2}-N_{21} E_{1}-N_{22} E_{2}
$$

Using the properties of the generalized matrix inverse [17] (50) has a solution for $\left[\begin{array}{ll}E_{2} & K_{2}\end{array}\right]$ iff

$$
\left[\begin{array}{ll}
L_{2} \bar{A} & L_{2} D
\end{array}\right]\left(I-\Sigma^{+} \Sigma\right)=0
$$


or equivalently,

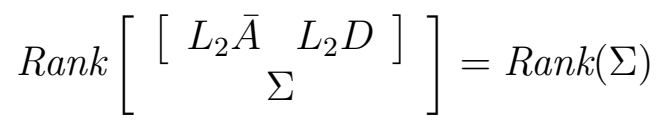

In the case that (50) has a solution, the solution is given by

$$
\left[\begin{array}{ll}
E_{2} & K_{2}
\end{array}\right]=\left[\begin{array}{ll}
L_{2} \bar{A} & L_{2} D
\end{array}\right] \Sigma^{+}+Z_{2}\left(I-\Sigma \Sigma^{+}\right)
$$

where $Z_{2}$ is any arbitrary matrix of appropriate dimension. Using (54) in (48) and (49), the following can be written

$$
\begin{aligned}
& N_{21}=F_{21}-Z_{2} G_{1} \\
& N_{22}=F_{22}-Z_{2} G_{2}
\end{aligned}
$$

where

$$
\begin{aligned}
& F_{21}=\left[L_{2} A M_{1}\right]-\left[\begin{array}{ll}
L_{2} \bar{A} & L_{2} D
\end{array}\right] \Sigma^{+}\left[\begin{array}{c}
C A M_{1} \\
C M_{1}
\end{array}\right] \\
& F_{22}=\left[\begin{array}{ll}
L_{2} A M_{2}
\end{array}\right]-\left[\begin{array}{ll}
L_{2} \bar{A} & L_{2} D
\end{array}\right] \Sigma^{+}\left[\begin{array}{c}
C A M_{2} \\
C M_{2}
\end{array}\right]
\end{aligned}
$$

Remark 2: The observer matrices, $E_{1}, N_{11}, N_{12} J_{1}, H_{1}$, can be computed using (40), (33), (34), (36) and (27) respectively. The other observer matrices $E_{2}, N_{21}, N_{22}, J_{2}, H_{2}$ exist if and only if (53) is satisfied and can be computed using (54), (55), (56), (51) and (28) respectively. The existence of the observer (4)-(6), i.e. finding solutions for all of the observer parameters, $E_{1}, E_{2}, J_{1}, J_{2}, H_{1}, H_{2}, N_{11}, N_{12}, N_{21}$ and $N_{22}$, depends on the satisfaction of (53) which is also presented in the following theorem

Theorem 2: The observer of the form (4)-(6) exists iff

$$
\operatorname{Rank}\left[\begin{array}{cc}
L_{2} A & L_{2} D \\
C A & C D \\
C & 0 \\
L_{2} & 0
\end{array}\right]=\operatorname{Rank}\left[\begin{array}{cc}
C A & C D \\
C & 0 \\
L_{2} & 0
\end{array}\right]
$$


Proof:

To prove (59) consider the $L H S$ of (59)

$$
\begin{aligned}
\operatorname{Rank}\left[\begin{array}{cc}
L_{2} A & L_{2} D \\
C A & C D \\
C & 0 \\
L_{2} & 0
\end{array}\right]=\operatorname{Rank}\left[\begin{array}{cc}
L_{2} A & L_{2} D \\
C A & C D \\
C & 0 \\
L_{2} & 0
\end{array}\right]\left[\begin{array}{ccc}
M_{2} & I-M_{2} L_{2} & 0 \\
0 & 0 & I_{q}
\end{array}\right] \\
=\operatorname{Rank}\left[\begin{array}{ccc}
L_{2} A M_{2} & L_{2} A\left(I-M_{2} L_{2}\right) & L_{2} D \\
C A M_{2} & C A\left(I-M_{2} L_{2}\right) & C D \\
C M_{2} & C\left(I-M_{2} L_{2}\right) & 0 \\
L_{2} M_{2} & L_{2}\left(I-M_{2} L_{2}\right) & 0
\end{array}\right] \\
=\operatorname{Rank}\left[\begin{array}{ccc}
L_{2} A M_{2} & L_{2} \bar{A} & L_{2} D \\
C A M_{2} & C \bar{A} & C D \\
C M_{2} & \bar{C} & 0 \\
I & 0 & 0
\end{array}\right] \\
\therefore \text { LHS }=r+\operatorname{Rank}\left[\begin{array}{cc}
{\left[L_{2} \bar{A}\right.} & \left.L_{2} D\right] \\
\Sigma & \Sigma
\end{array}\right]
\end{aligned}
$$

Now consider the RHS of (59)

$$
\begin{aligned}
\operatorname{Rank}\left[\begin{array}{cc}
C A & C D \\
C & 0 \\
L_{2} & 0
\end{array}\right] & =\operatorname{Rank}\left[\begin{array}{cc}
C A & C D \\
C & 0 \\
L_{2} & 0
\end{array}\right]\left[\begin{array}{ccc}
M_{2} & I-M_{2} L_{2} & 0 \\
0 & 0 & I_{q}
\end{array}\right] \\
& =\operatorname{Rank}\left[\begin{array}{ccc}
C A M_{2} & C \bar{A} & C D \\
C M_{2} & \bar{C} & 0 \\
I & 0 & 0
\end{array}\right] \\
\therefore R H S & =r+\operatorname{Rank}(\Sigma)
\end{aligned}
$$

Given (60) and (61), (53) will be satisfied if and only if (59) holds.

Theorem 2 ensures the solvability of the observer parameters $N, J, H$, and $E$. However, it does not ensure that the matrix $N_{22}$ is Hurwitz. It can be seen from (56) that $N_{22}$ is stable if and only if the pair $\left(F_{22}, G_{2}\right)$ is detectable. The necessary and sufficient condition for $N_{22}$ to be Hurwitz is given in the following theorem. 
Theorem 3: The matrix $N_{22}$ is Hurwitz iff $\operatorname{Rank}\left[\begin{array}{cc}s L_{2}-L_{2} A & -L_{2} D \\ C A & C D \\ C & 0\end{array}\right]=\operatorname{Rank}\left[\begin{array}{cc}C A & C D \\ C & 0 \\ L_{2} & 0\end{array}\right] \forall s \in \mathbb{C}: \operatorname{Re}(s) \geq 0$

\section{Proof:}

Consider the LHS of (62)

$$
\begin{aligned}
& =\operatorname{Rank}\left[\begin{array}{cc}
s L_{2}-L_{2} A & -L_{2} D \\
C A & C D \\
C & 0
\end{array}\right] \\
& =\operatorname{Rank}\left[\begin{array}{cc}
s L_{2}-L_{2} A & -L_{2} D \\
C A & C D \\
C & 0
\end{array}\right]\left[\begin{array}{ccc}
M_{2} & I-M_{2} L_{2} & 0 \\
0 & 0 & I_{q}
\end{array}\right] \\
& =\operatorname{Rank}\left[\begin{array}{ccc}
s L_{2} M_{2}-L_{2} A M_{2} & -L_{2} \bar{A} & -L_{2} D \\
C A M_{2} & C \bar{A} & C D \\
C M_{2} & \bar{C} & 0
\end{array}\right] \\
& =\operatorname{Rank}\left[\begin{array}{cc}
s I-L_{2} A M_{2} & {\left[\begin{array}{cc}
-L_{2} \bar{A} & -L_{2} D
\end{array}\right]} \\
{\left[\begin{array}{c}
C M_{2} \\
C M_{2}
\end{array}\right]} & \Sigma
\end{array}\right]
\end{aligned}
$$

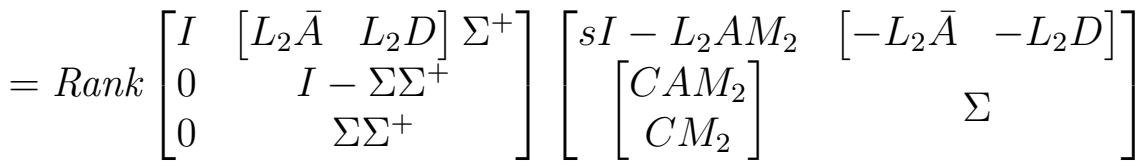

$$
\begin{aligned}
& \operatorname{Rank}\left[\begin{array}{cc}
s I-F_{22} & 0 \\
G_{2} & 0 \\
\Sigma \Sigma^{+}\left[\begin{array}{c}
C A M_{2} \\
C M_{2}
\end{array}\right] \Sigma
\end{array}\right] \\
& \operatorname{Rank}\left[\begin{array}{cc}
s I-F_{22} & 0 \\
G_{2} & 0 \\
\Sigma \Sigma^{+}\left[\begin{array}{c}
C A M_{2} \\
C M_{2}
\end{array}\right] \Sigma
\end{array}\right]\left[\Sigma^{+}\left[\begin{array}{cc}
I & 0 \\
C A M_{2} \\
C M_{2}
\end{array}\right] \quad I\right] \\
& =\operatorname{Rank}\left[\begin{array}{c}
s I-F_{22} \\
G_{2}
\end{array}\right]+\operatorname{Rank}(\Sigma)
\end{aligned}
$$


$\therefore L H S=r+\operatorname{Rank}(\Sigma), \forall s \in \mathbb{C}: \operatorname{Re}(s) \geq 0$.

From (56) and (62) if follows that, if and only if $(62)$ is satisfied $\left(F_{22}, G_{2}\right)$ is detectable.

Remark 3: As argued in [11], during sliding motion $\dot{e}_{y}=e_{y}=0$ and therefore (22) can be rewritten as

$$
\dot{e}_{1}=N_{22} e_{1}
$$

and in order to sustain sliding motion, and for (asymptotic) state estimation error decay, the eigenvalues of $N_{22}$ must be stable. So, satisfaction of (62) is a necessary condition for sliding to occur.

In the following we present the design algorithm for the sliding mode functional observer.

\section{Design Algorithm}

1. Partition $L$ according to $L=\left[\begin{array}{c}L_{1} \\ L_{2}\end{array}\right]=\left[\begin{array}{c}G C \\ L_{2}\end{array}\right]$

2. Check if condition (59) is satisfied, if yes continue, otherwise a sliding mode functional observer doesn't exist.

3. Compute $F_{22}$ using (58), $G_{2}$ using (46) and check if the pair $\left(F_{22}, G_{2}\right)$ is detectable. If yes continue, otherwise $N_{22}$ cannot be made Hurwitz and consequently a stable sliding mode functional observer doesn't exist.

4. Using (42) and any poleplacement method obtain $Z_{2}$ to make $N_{22}$ Hurwitz.

5. Use (57) to compute $F_{21}$, compute $G_{1}$ using (45) and then use (55) to compute $N_{21}$.

6. Choose any $Z_{1}$ and use (41) and (42) to compute $N_{11}$ and $N_{12}$ respectively.

7. Use (40) and (54) compute $E_{1}, K_{1}, E_{2}$ and $K_{2}$.

8. Use (36) and (51) to compute $J_{1}$ and $J_{2}$ respectively.

9. Compute $P_{1}$ and $P_{2}$ using (25) and (26) respectively.

10. Compute $H_{1}$ and $H_{2}$ according to (25) and (28) respectively.

11. Use any standard sliding mode technique [2], [18] to obtain $\Gamma_{1}$ to make the error dynamics of (21) stable.

The sliding mode functional observer can be simplified if $L=I_{n}$ and $D=0_{n \times q}$. In this case both Theorem 2 and 3 are satisfied, and the observer structure (4)-(6) proposed in this paper will in fact reduce to the Utkin 
Observer [2] and the design algorithm proposed in this paper reduces to design algorithm of the Utkin Observer. If however, $L=I_{n}$ and $D \neq 0$ and sliding is not considered (i.e. $L_{2}=L$ ) then the necessary and sufficient conditions proposed in this paper reduce to the well known matching and observability conditions which are necessary and sufficient for the design of unknown input observers. In particular, Theorem 2 reduces to

$$
\operatorname{Rank}(C D)=\operatorname{Rank}(D)
$$

which is referred to as the matching condition as described in the literature [13] and Theorem 3 reduces to

$$
\operatorname{Rank}\left[\begin{array}{cc}
s I_{n}-A & D \\
C & 0
\end{array}\right]=n+m, \forall s \in \mathbb{C}: \operatorname{Re}(s) \geq 0
$$

which is referred to as the observability condition for unknown input observers as seen in [15] and [16].

Remark 4: If the sliding mode functional observer existence conditions as detailed in the design algorithm can be satisfied then a sliding mode observer of order $r<n$ can be designed. However, if those sliding mode observer existence conditions cannot be satisfied then the required linear functions $z(t)$ can still be estimated by designing an $n$ order Utkin observer to estimate all the individual states and then linearly combine those to obtain the required $z(t)$. The order of the Utkin observer is obviously greater than the order of a sliding mode functional observer.

\section{Application to Speed Sensorless Control of Permanent Magnet Synchronous Machines}

Speed sensorless control of electrical machines is an area where the importance of lower order, robust estimation algorithms that are insensitive to machine parameter variations is highlighted [19]. In order to demonstrate the effectiveness of the Sliding Mode Functional Observer presented in this paper, we now use its application for speed sensorless control of a surface mounted Permanent Magnet Synchronous Motor (PMSM). It must be mentioned here that there has been reported work on application of sliding mode technique for sensorless control of PMSMs earlier [20]. However, the sliding technique presented in this paper is novel and hence its usage for sensorless control of PMSMs. With the observer topology presented in this paper, 
there exists the added advantage of estimating the load torque or such other linear functions of the state variables in addition to the rotor speed, which is not a feature in most of the sensorless control algorithms found in literature [19]. Another motivation to use this Sliding Mode Functional Observer is its estimation strategy. Most of the speed sensorless control algorithms for PMSMs can be classified into two categories. i.e. Back emf based methods and high frequency injection type methods. It is well-known that the former is more applicable in surface mounted type PMSMs [21], while the latter can be used only with interior magnet type PMSMs because of the difference in direct and quadrature axis self inductances [22]. One major problem in back emf based methods is their poor performance in low speeds due to low signal to noise ratio in the sampled back emf [21]. The sliding technique proposed in this paper is based on the quadrature axis current estimation error and therefore this method is capable of estimating the rotor speed even at low speed region. With this background, the formation of the standard dynamical model of the surface mounted PMSM into a system with unknown input will be demonstrated in the following sub-sections.

\subsection{Dynamical Model of PMSM}

The dynamical model of a Permanent Magnet Synchronous Motor (PMSM) in synchronous reference frame can be given as

$$
\begin{aligned}
\frac{d i_{d}(t)}{d t} & =-\frac{R_{s}}{L_{d}} i_{d}(t)+\frac{\omega L_{q}}{L_{d}} i_{q}(t)+\frac{1}{L_{d}} u_{d}(t) \\
\frac{d i_{q}(t)}{d t} & =-\frac{R_{s}}{L_{q}} i_{q}(t)+\frac{\omega L_{d}}{L_{q}} i_{d}(t)-\frac{\omega \psi_{m}}{L_{q}}+\frac{1}{L_{q}} u_{q}(t) \\
T_{m} & =\frac{3}{2} n_{p}\left[\psi_{m} i_{q}(t)-\left(L_{q}-L_{d}\right) i_{d}(t) i_{q}(t)\right) \\
\frac{d \omega}{d t} & =\frac{1}{J_{m}}\left[T_{m}-T_{l}-B_{m} \omega\right],
\end{aligned}
$$

where $i_{d}(t)=$ Direct axis current, $i_{q}(t)=$ Quadrature axis current, $R_{s}=$ Stator resistance, $L_{d}=$ Direct axis self inductance, $L_{q}=$ Quadrature axis self inductance, $\omega=$ Rotor electrical angular speed, $u_{d}(t)=$ Direct axis input voltage, $u_{q}(t)=$ Quadrature axis input voltage, $T_{m}=$ Motor torque, $n_{p}=$ Pole pair number, $\psi_{m}=$ Flux due to permanent magnets in air gap, $J_{m}=$ Rotor inertia, $T_{l}=$ Load torque, and $B_{m}=$ Torque due to damping [23]. 
Now we will first convert the dynamical equations of PMSM in (66) into a linear state space form. Being time varying quantities under normal circumstances, the rotor electrical angular speed $\omega$, motor torque $T_{m}$ and load torque $T_{l}$ can also be rewritten with time argument and the terms in (66) can first be reorganized as:

$$
\begin{array}{r}
\frac{d i_{d}(t)}{d t}=-\frac{R_{s}}{L_{d}} i_{d}(t)+\frac{L_{q}}{L_{d}} \omega(t) i_{q}(t)+\frac{1}{L_{d}} u_{d}(t) \\
\frac{d i_{q}(t)}{d t}=-\frac{L_{d}}{L_{q}} \omega(t) i_{d}(t)-\frac{R_{s}}{L_{q}} i_{q}(t)-\frac{\psi_{m}}{L_{q}} \omega(t)+\frac{1}{L_{q}} u_{q}(t) \\
0=\frac{3}{2} n_{p} \psi_{m} i_{q}(t)-\frac{3}{2} n_{p} \psi_{m}\left(L_{q}-L_{d}\right) i_{d}(t) i_{q}(t)-T_{m}(t) \\
\frac{d \omega(t)}{d t}=-\frac{B_{m}}{J_{m}} \omega(t)+\frac{1}{J_{m}} T_{m}(t)-\frac{1}{J_{m}} T_{l}(t),
\end{array}
$$

It can now be seen that the terms $+\frac{L_{q}}{L_{d}} \omega(t) i_{q}(t),-\frac{L_{d}}{L_{q}} \omega(t) i_{d}(t)$, and $-\frac{3}{2} n_{p} \psi_{m}\left(L_{q}-L_{d}\right) i_{d}(t) i_{q}(t)$ in $(70),(71)$ and (72) introduce non-linear terms into the state space model. If we apply the well-known axis decoupling [23] by introducing a new control input defined by

$$
\begin{aligned}
& u_{d}(t)=u_{d}^{\prime}(t)-L_{q} \omega(t) i_{q}(t) \\
& u_{q}(t)=u_{q}^{\prime}(t)+L_{d} \omega(t) i_{d}(t),
\end{aligned}
$$

nonlinear terms in (70) and (71) will vanish. In case of surface mounted PMSMs, where $L_{d} \simeq L_{q}$, nonlinear term in (72) can be omitted [23]. These steps would result in

$$
\begin{aligned}
\frac{d i_{d}(t)}{d t} & =-\frac{R_{s}}{L_{d}} i_{d}(t)+\frac{1}{L_{d}} u_{d}^{\prime}(t) \\
\frac{d i_{q}(t)}{d t} & =-\frac{R_{s}}{L_{q}} i_{q}(t)-\frac{\psi_{m}}{L_{q}} \omega(t)+\frac{1}{L_{q}} u_{q}^{\prime}(t) \\
\frac{d \omega(t)}{d t} & =-\frac{B_{m}}{J_{m}} \omega(t)+\frac{1}{J_{m}} T_{m}(t)-\frac{1}{J_{m}} T_{l}(t), \\
0 & =\frac{3}{2} n_{p} \psi_{m} i_{q}(t)-T_{m}(t)
\end{aligned}
$$

\subsection{Conversion of PMSM model into a System with Unknown Input}

In this approach $T_{m}(t)$ is eliminated from the (76) by substituting for it using (79). Then, if the load torque $T_{l}(t)$, which usually is treated as a 
disturbance in dynamical modeling of electrical machines, is considered as an unknown input, it can now be seen that the dynamical model of a surface mounted PMSM in synchronous reference frame can be given as:

$$
\begin{aligned}
{\left[\begin{array}{c}
\dot{i}_{d}(t) \\
\dot{i}_{q}(t) \\
\dot{\omega}(t)
\end{array}\right]=} & {\left[\begin{array}{ccc}
-\frac{R_{s}}{L_{d}} & 0 & 0 \\
0 & -\frac{R_{s}}{L_{q}} & -\frac{\psi_{m}}{L_{q}} \\
0 & \frac{3}{2 J_{m}} n_{p} \psi_{m} & -\frac{B_{m}}{J_{m}}
\end{array}\right]\left[\begin{array}{c}
i_{d}(t) \\
i_{q}(t) \\
\omega(t)
\end{array}\right] } \\
& +\left[\begin{array}{cc}
\frac{1}{L_{d}} & 0 \\
0 & \frac{1}{L_{q}} \\
0 & 0
\end{array}\right]\left[\begin{array}{c}
u_{d}^{\prime}(t) \\
u_{q}^{\prime}(t)
\end{array}\right]+\left[\begin{array}{c}
0 \\
0 \\
-\frac{1}{J_{m}}
\end{array}\right] T_{l}(t) .
\end{aligned}
$$

If the problem under consideration is designing an observer for the estimation of rotor speed for implementation of sensorless control, then the typical output equation will be

$$
y(t)=\left[\begin{array}{lll}
1 & 0 & 0 \\
0 & 1 & 0
\end{array}\right]\left[\begin{array}{c}
i_{d}(t) \\
i_{q}(t) \\
\omega(t)
\end{array}\right] .
$$

Now with the substitutions

$$
\begin{aligned}
& A=\left[\begin{array}{ccc}
-\frac{R_{s}}{L_{d}} & 0 & 0 \\
0 & -\frac{R_{s}}{L_{q}} & -\frac{\psi_{m}}{L_{q}} \\
0 & \frac{3}{2 J_{m}} n_{p} \psi_{m} & -\frac{B_{m}}{J_{m}}
\end{array}\right], B=\left[\begin{array}{cc}
\frac{1}{L_{d}} & 0 \\
0 & \frac{1}{L_{q}} \\
0 & 0
\end{array}\right], \\
& D=\left[\begin{array}{c}
n_{d} \\
n_{q} \\
-\frac{1}{J_{m}}
\end{array}\right] \text { and } C=\left[\begin{array}{lll}
1 & 0 & 0 \\
0 & 1 & 0
\end{array}\right] \text { together with } x(t)=\left[\begin{array}{c}
i_{d}(t) \\
i_{q}(t) \\
\omega(t)
\end{array}\right], u(t)= \\
& {\left[\begin{array}{c}
u_{d}^{\prime}(t) \\
u_{q}^{\prime}(t)
\end{array}\right] \text { and } v(t)=T_{l}(t),}
\end{aligned}
$$

(82) and (83) can be expressed in the form of equations (1), (2) and (3). $n_{d}, n_{q}$ in $D$ above represent the measurement noise variance and possible drifts introduced by the current sensors to the sampled $i_{d}(t)$ and $i_{q}(t)$ currents. The sign and magnitude of $n_{d}$ and $n_{q}$ must be determined based on the frequency domain analysis of sampled currents and the operating load torque range of the drive. 


\begin{tabular}{||l|c||}
\hline Parameter & Value \\
\hline Rated voltage / VAC & 230 \\
\hline Induced emf / Vrms,$l-l / 1000 \mathrm{rpm}$ & 42 \\
\hline Rated current / A & 16.3 \\
\hline Rated torque / Nm & 10.5 \\
\hline Rated speed / rpm & 3000 \\
\hline Number of poles & 6 \\
\hline Rotor inertia / $\mathrm{kgm}^{2}$ & 0.00167 \\
\hline Stator resistance / $\Omega_{l-l}$ & 0.33 \\
\hline Inductance / $\mathrm{mH}_{l-l}$ & 3.2 \\
\hline
\end{tabular}

Table 1: Machine Parameter Values

\begin{tabular}{||l|c||}
\hline Primary Base Quantity & Base value \\
\hline Voltage $\left(U_{\text {base }}\right)$ & $103 \mathrm{~V}$ \\
\hline Current $\left(I_{\text {base }}\right)$ & $23.1 \mathrm{~A}$ \\
\hline Electrical angular speed $\left(\omega_{\text {base }}\right)$ & $942 \mathrm{rad} / \mathrm{s}$ \\
\hline Time $\left(t_{\text {base }}\right)$ & $0.00106 \mathrm{~s} / \mathrm{rad}$ \\
\hline
\end{tabular}

Table 2: Machine Base Values

\subsection{PMSM Test Rig}

Two back-to-back connected PMSM machines (one to be operated as the drive, while the other to be controlled as the load for load tests) was used for the validation of performance of the observer. Each motor has the specifications given in Table 1 . The design is done using the per-unit quantities calculated with respect to the base quantities given in Table 2 . Thus the per-unit values of $R_{s}, L_{d}, L_{q}, \psi_{m}, J_{m}$ and $B_{m}$ are given in Table 3.

\subsection{Design of Sliding Mode Functional Observer}

From above per-unit values of the PMSM drive, system matrices $A, B$, $C$ and $D$ computed will be:

$$
A=\left[\begin{array}{rrr}
-0.1121 & 0 & 0 \\
0 & -0.1057 & -2.8571 \\
0 & 0.0412 & -0.0011
\end{array}\right]
$$




\begin{tabular}{||c|c||}
\hline Motor Parameter & Per-unit value \\
\hline$R_{s}$ & 0.037 \\
\hline$L_{d}$ & 0.033 \\
\hline$L_{q}$ & 0.035 \\
\hline$\psi_{m}$ & 1 \\
\hline$J_{m}$ & 109.3 \\
\hline$B_{m}$ & 0.1256 \\
\hline
\end{tabular}

Table 3: Machine Parameters in Per-unit

$B=\left[\begin{array}{cc}3.0303 & 0 \\ 0 & 2.8571 \\ 0 & 0\end{array}\right], C=\left[\begin{array}{lll}1 & 0 & 0 \\ 0 & 1 & 0\end{array}\right]$

and with $n_{d}=n_{q}=0.0001, D=\left[\begin{array}{c}0.0001 \\ 0.0001 \\ -0.0091\end{array}\right]$. Let us assume that we want

to estimate the rotor electrical speed. Hence the function to be estimated given by

$$
L=\left[\begin{array}{lll}
0 & 1 & 0 \\
0 & 0 & 1
\end{array}\right]
$$

This means that the sliding mode is implemented using the estimated qaxis current $\hat{i}_{q}(t)$. It is well known that in controlling the surface mounted PMSMs, the d-axis current, which is the flux producing current is controlled at zero (since the air gap flux is provided by the permanent magnets). Therefore, in order to estimate the speed, sliding surface has to be created around the q-axis current. This will result in a reduced order observer which uses only one current information. To obtain the sliding surface for the observer, $G$ is obtained according to (10)

$$
G=\left[\begin{array}{ll}
0 & 1
\end{array}\right] .
$$

and the sliding surface according to (12) is given below

$$
M e(t)=\left[\begin{array}{ll}
0 & 1
\end{array}\right] y(t)-\left[\begin{array}{ll}
1 & 0
\end{array}\right] \hat{z}(t) .
$$

It is easy to verify that condition (59) is satisfied. Now matrix $F_{22}$ and $G_{2}$ can be obtained according to (58) and (46) and is given below

$F_{22}=[-396.0052]$ 
and $G_{2}=\left[\begin{array}{llll}3.9109 & -3.9109 & 0.4385 & 0\end{array}\right]^{T}$.

Since the pair $\left(F_{22}, G_{2}\right)$ is detectable, $N_{22}$ can be made Hurwitz and its pole is chosen to be $\{-0.15\}$ (justification for this pole placement will be given in the following section $E$ ). Using (56) $Z_{2}$ and $N_{22}$ can be obtained as follows

$Z_{2}=\left[\begin{array}{llll}-50.2934 & 50.2934 & -5.6390 & 0\end{array}\right]$ and

$N_{22}=[-0.15]$

Using (57) and (45) we can find $F_{21}$ and $G_{1}$ respectively as follows

$F_{21}=[-4.2269]$ and

$G_{1}=\left[\begin{array}{llll}0.043 & -0.043 & 0.0048 & 1.0\end{array}\right]^{T}$.

Using (48) we can compute $N_{21}$ as follows

$N_{21}=[0.1231]$

The choice of $Z_{1}$ is arbitrary and we choose

$Z_{1}=\left[\begin{array}{llll}0 & 0 & 0 & 0\end{array}\right]$

We can now compute $N_{11}$ and $N_{12}$ using (41) and (42) as follows

$N_{11}=[-0.0430]$ and $N_{12}=[-3.9109]$.

Using (40) and (54) we can compute $E$ and $K$ as follows

$E=\left[\begin{array}{cc}0.4969 & 0.5031 \\ -99.9811 & -0.0189\end{array}\right]$ and $K=\left[\begin{array}{cc}0.0557 & 0 \\ -11.2100 & 0\end{array}\right]$.

Matrix $P$ can be computed from (25) and (26), $H$ from (27) and (28) and $J$ from (36) and (51) as follows

$$
\begin{aligned}
& P=\left[\begin{array}{ccc}
-0.4969 & 0.4969 & 0 \\
99.9811 & 0.0189 & 1.0
\end{array}\right] \\
& H=\left[\begin{array}{cc}
-1.6730 & 1.2906 \\
336.6367 & 0.0491
\end{array}\right]
\end{aligned}
$$


and

$J=\left[\begin{array}{cc}391.0480 & 0.0523 \\ 3.8483 & 0.0648\end{array}\right]$.

The error dynamics of the observer can be written as follows (note that $e_{y}(t) \in \mathbb{R}$ and $\left.e_{1}(t) \in \mathbb{R}\right)$

$$
\begin{aligned}
{\left[\begin{array}{c}
\dot{e}_{y}(t) \\
\dot{e}_{1}(t)
\end{array}\right]=} & {\left[\begin{array}{cc}
-0.0430 & -3.9109 \\
0.1231 & -0.1500
\end{array}\right]\left[\begin{array}{l}
e_{y}(t) \\
e_{1}(t)
\end{array}\right] } \\
& -\left[\begin{array}{c}
\Gamma_{1} \operatorname{sgn}\left(e_{y}(t)\right) \\
0
\end{array}\right]
\end{aligned}
$$

\subsection{Simulation of Sliding Mode Functional Observer}

In order to verify the performance of the Sliding Model Functional Observer designed, a simulation study was carried out. During this study, the PMSM model was controlled in closed loop using the measured $d$ and $q$ axis currents and estimated speed as feedbacks. The observer was used for estimation of the speed so that its convergence properties could be verified under different operating conditions. The Fig. 1 below shows the complete simulation model in block diagram form. Simulations were carried out using Matlab ${ }^{\circledR} / S I M U L I N K^{\circledR}$ platform. In this section, the strength of the proposed sliding mode functional observer will be demonstrated by simulations showing; convergence properties with initial estimation errors, closed loop speed sensorless operation, estimation under a step load torque disturbance and estimation with inaccurate machine parameters (parameter sensitivity). First, some guidelines will be given on tuning the current controller, observer pole, sliding mode gain and the speed controller.

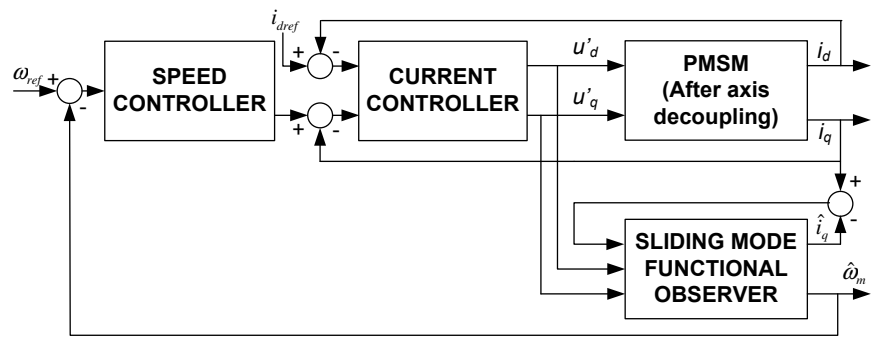

Figure 1: Complete simulation model in block diagram form 


\subsubsection{Guidelines for Tuning}

In this type of a sensorless control algorithm, the estimation of the speed is dependant on the speed information contained in the sampled motor currents. Then the closed loop speed controller is dependant on the estimated speed by the observer. As such a rule of thumb in fixing the closed loop bandwidth of current controller $(\alpha)$, pole placement of sliding mode observer $(p)$ and closed loop bandwidth of speed controller $\left(\alpha_{s}\right)$ must be according to in inequality $\alpha>p>\alpha_{s}$ [23]. It is recommended to make each pair of bandwidths at least a decade apart. As such, for these simulations the values chosen in per unit domain are: $\alpha=2, p=-0.15$ and $\alpha_{s}=0.02$. These settings lead to a closed loop rise time of $d$-axis and $q$-axis currents of approximately $1-2 \mathrm{~ms}$ and a closed loop speed rise time of approximately $100 \mathrm{~ms}$ with an overshoot of $10 \%$. It must be noted here that these settings are without using any integrator anti-windup mechanism for the current and speed controllers and both control loops can be made faster by incorporating a suitable integrator anti-windup algorithm [23]. Fixing sliding mode gain $\left(\Gamma_{1}\right)$ must be with respect to the bandwidth of the observer (i.e. pole placement) and it is recommended to have a value in the neighborhood of $1 \%$ of $p$. The value used in these simulations is $\Gamma_{1}=0.0001$.

\subsubsection{Convergence Properties with Initial Estimation Errors}

In sensorless control of PMSM, startup is always a challenging problem. The reason is not knowing the exact rotor position information. This is a separate research area and there is several published work on startup techniques of PMSMs [23]. As far as the performance of this particular algorithm is concerned, it is important to demonstrate its convergence capability with initial estimation errors in $q$-axis current and speed $\left(e_{y}(0)\right.$ and $e_{1}(0)$ respectively). The Fig 2. shows the error response, when $\left(e_{y}(0)=0.005\right)$ and $\left(e_{1}(0)=0.0055\right)$. It can be seen that sliding occurs on the plane $e_{y}(t)=0$. As can be seen in Fig 2., the initial error in $e_{y}(t)$ is dragged towards the plane $e_{y}(t)=0$ in finite time and $e(t)$ slides along the plane $e_{y}(t)=0$ towards the origin.

\subsubsection{Closed Loop Speed Sensorless Operation}

The speed control loop is closed by feeding back the estimated speed from the sliding mode observer and a step reference input of $1 \mathrm{pu}$ is applied to the system at $t=0$. The resulting $q$-axis current variation and the speed 


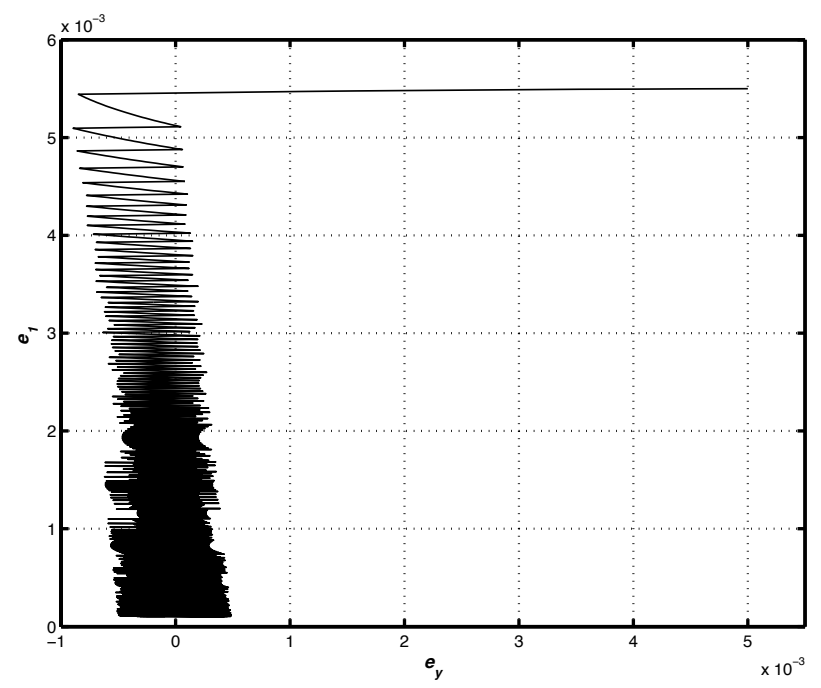

Figure 2: Error Trajectory for $e_{y}$ and $e_{1}$

response is shown in Fig. 3. Time variations of $e_{y}(t)$ and $e_{1}(t)$ during the transient period are shown in Fig. 4 (only the first 200ms is shown).
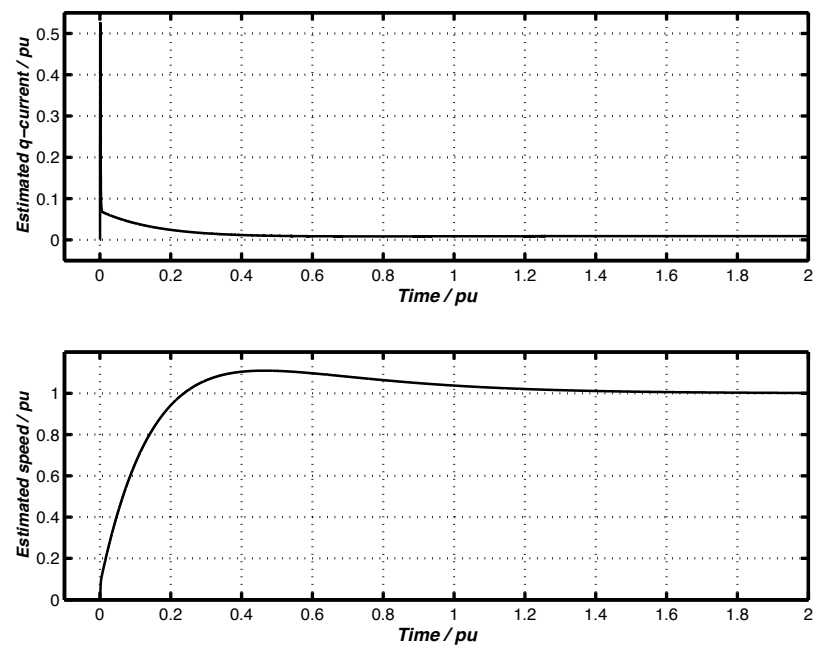

Figure 3: $q$-axis current variation and the speed response for a step

\subsubsection{Estimation Under a Step Load Torque Disturbance}

After the initial $1 \mathrm{pu}$ speed step is applied and the PMSM has reached closer to the steady state, a step load torque of $0.2 \mathrm{pu}$ is applied at $t=1.5$ 

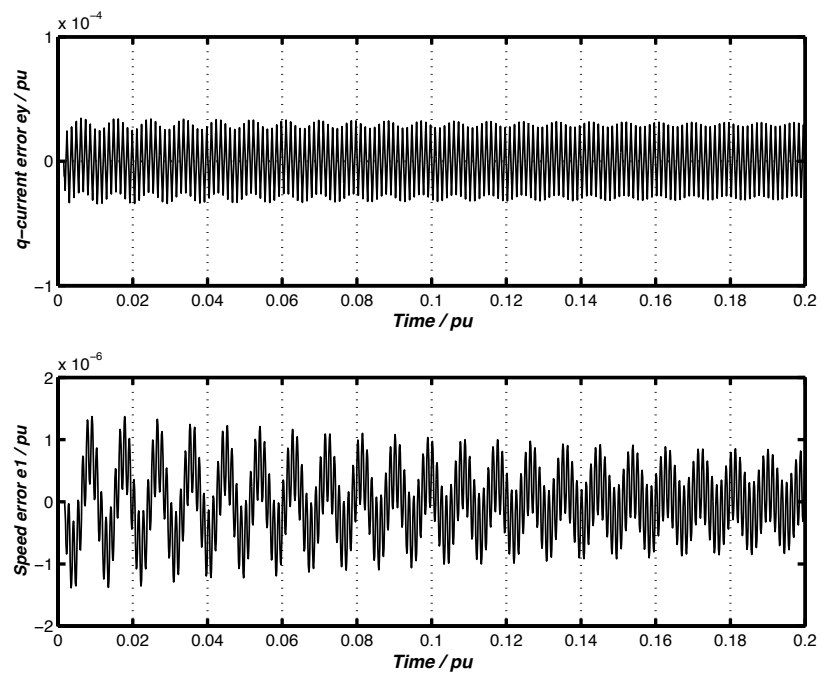

Figure 4: Time variations of $e_{y}(t)$ and $e_{1}(t)$ during the transient period

s. Since Proportional Integral control is used in the speed loop, motor speed gradually recovers after this load disturbance back to the set speed $0.1 \mathrm{pu}$. In Fig. 5 shown are the $q$-axis current variation and the speed response. Time variations of $e_{y}(t)$ and $e_{1}(t)$ during the speed fluctuation are shown in Fig. 6. It must be noted here that the saw-tooth nature of $e_{y}(t)$ is due to the
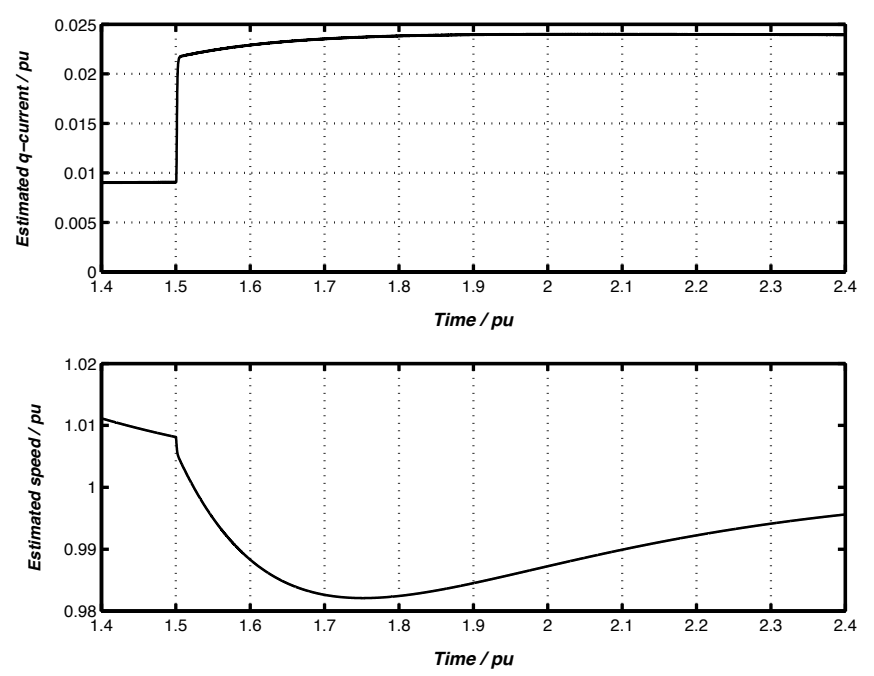

Figure 5: $q$-axis current and the speed response for a step load torque 

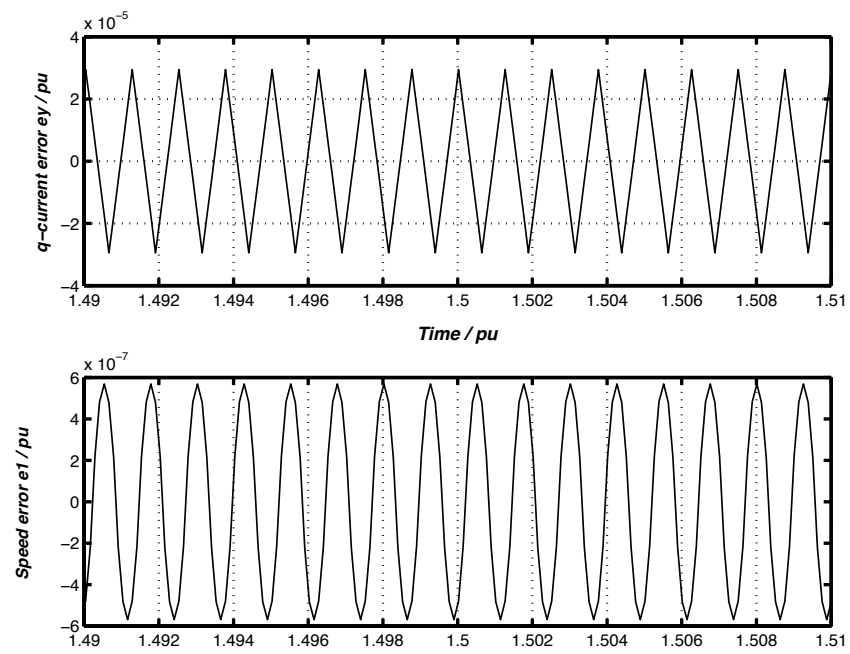

Figure 6: Time variations of $e_{y}(t)$ and $e_{1}(t)$ during the speed fluctuation

sliding mode term in the error dynamics. Though some oscillatory behavior can be seen in $e_{1}(t)$, it must be noted that it is of negligible peak-to-peak amplitude. Both $e_{y}(t)$ and $e_{1}(t)$ do not show any significant change due to the load torque disturbance. This further establishes the robustness of the proposed observer.

\subsubsection{Estimation with Inaccurate Machine Parameters (Parameter Sensitiv- ity)}

Sensitivity to changing machine parameters is considered as one of the biggest challenges in developing reliable speed sensorless control algorithms for electrical machines [19], [23]. The $d$ and $q$ axes inductances and the stator resistance tend to change with the internal temperature of the machine. Aging can also cause changes in these quantities. As such, there can always be a difference between the actual machine parameters and the machine parameter values used in the PMSM model that is incorporated in designing the sliding mode observer. In order to verify the robustness of the proposed observer against machine parameter variations, $\pm 10 \%$ deviations are introduced to $L_{d}, L_{q}$ and $R_{s}$ used in the observer model with respect to those used in the simulated PMSM model. The Fig. 7 show the $q$-axis current variation and the speed response, when a step change in speed reference is applied. Time variations of $e_{y}(t)$ and $e_{1}(t)$ during the transient are shown in Fig. 8 
(only the first $200 \mathrm{~ms}$ is shown). It can be seen that the estimated $q$-axis
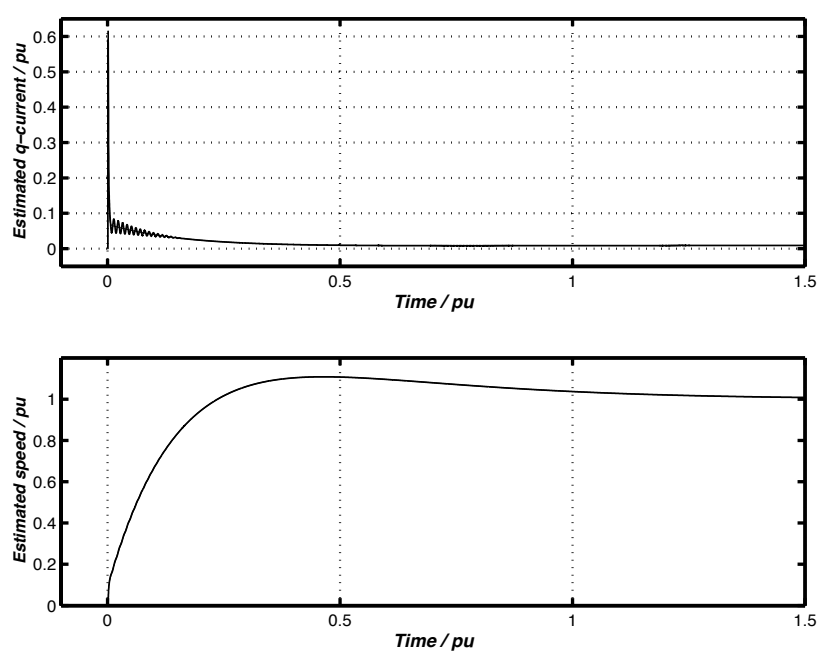

Figure 7: Estimated q-axis current and speed response with inaccurate machine parameters
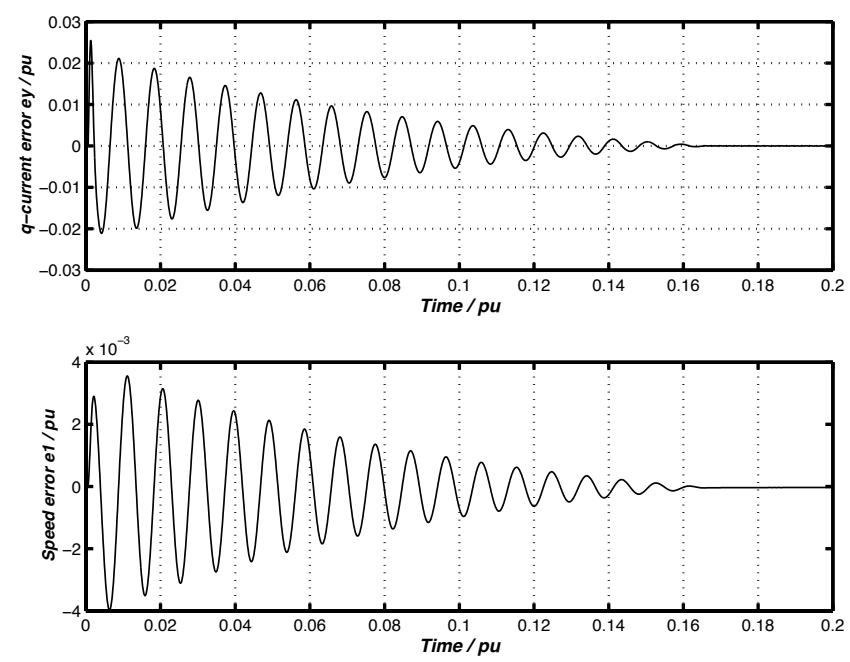

Figure 8: Time variations of $e_{y}(t)$ and $e_{1}(t)$ with inaccurate machine parameters

current heavily oscillates before showing the typical sliding mode switching after about $160 \mathrm{~ms}$. These oscillations do not make a significant impact on the closed loop control as measured $q$-axis current is used for feedback control. It 
can also be seen that the initial oscillations seen in the estimated speed is of negligible amplitude and hence, they do not make an impact when estimated speed is used for feedback control. Thus it can be seen that the proposed observer shows robust behavior even under parameter variations.

\section{Conclusion}

In this paper the problem of designing a Sliding Mode Functional Observer for a system with unknown inputs has been addressed. The conditions for the existence of such an observer are illustrated and proven. An eleven step design algorithm for the design of the sliding mode functional observer has been proposed. This theory has been applied for speed sensorless control of surface mounted PMSM.

\section{References}

[1] S.V. Drakunov, Sliding-Mode Observers Based on Equivalent Control Method, Proceedings of the 31st IEEE Conference on Decision and Control , Tucson, Arizona, December 16-18, pp. 2368- 2369, 1992.

[2] C. Edwards, and S.K. Spurgeon, Sliding Model Control: Theory and Applications, Taylor and Francis Ltd, U.K., 1998.

[3] K.D Young, V.I Utkin and U Ozguner, "A Control Engineer's Guide to Sliding Mode Control", IEEE Transactions on Control Systems Technology, vol. 7, pp.328-342, 1999.

[4] C.P. Tan, and C. Edwards, "An LMI Approach for Designing Sliding Mode Observers", IEEE Conference on Decision and Control, Australia, pp.2587-2592, 2000.

[5] V.I. Utkin, A Method of Separation of Motion for Designing a State Identifier, Problems of Control of multivariable Systems , Nauka, Moscow, 1983.

[6] V.I. Utkin, "Sliding Mode Control Design Principles and Applications to Electric Drives", IEEE Transactions on Industrial Electronics, vol.40, pp.23-36, 1993. 
[7] A. Hace, "Sliding Mode Control With Disturbance Observer for a Linear Belt Drive", IEEE Transactions on Industrial Electronics, vol.54, pp. 3042-3118, 2007.

[8] E.Bizkevelci, K.Leblebicioglu, H.B.Ertan, "A Sliding Mode Controller to Minimize SRM Torque Ripple and Noise", IEEE Transations on Industrial Electronics, vol.2 , pp.1333-1338 2004.

[9] M.A.Fnaiech, F.Betin, F.Fnaiech, G.A.Capolino, "Sliding Mode Control for Dual Three-phase Induction Motor Drives", IEEE Transactions on Industrial Electronics, vol. 2, pp. 2281-2285, 2006.

[10] C.Fallaha, M.Saad, H.Kanaan, W.H.Zhu, "Sliding Mode Nonlinear Switching Functions for Control Input Transient Constraints Reduction", IEEE Transactions on Industrial Electronics, vol. 2, pp.284-288 2006.

[11] C. Edwards, S.K. Spurgeon and C. P. Tan, "On the Development and Application of Sliding Mode Observers", Variable Structure Systems: Towards the 21st Century, LNCIS 274, pp. 253-282, 2002.

[12] J. F. Bejarano, L. Fridman, A.Poznyak, "Hierarchical second-order sliding-mode observer for linear time invariant systems with unknown inputs", International Journal of Systems Science, vol. 38, pp. 793-802, 2007.

[13] T. Floquet, C. Edwards, S. K. Spurgeon, "On sliding mode observers for systems with unknown inputs", International Journal of Adaptive Control and Signal Processing, vol. 21, pp. 638-656, 2007.

[14] T. Raff, F. Lachner and F. Allgower, "A Finite Unknown Input Observer for Linear Systems", IEEE Transactions on Automatic Control, pp.1-5, 2006

[15] P.Kudva, N. Viswanadham and A. Ramakrishna, "Observers for Linear Systems with Unknown Inputs, IEEE Transactions on Automatic Control, vol. AC-25, pp. 113-115, 1980.

[16] M.Hou and P.C Muller, "Design of Observers for Linear Systems with Unknown Inputs", IEEE Transactions on Automatic Control, vol. 37, pp. 871-875, 1992. 
[17] C.R. Rao and S.K. Mitra, "Generalized Inverse of Matrices and its Applications", New York, 1971.

[18] B. Bandyopadhyay, and S. Janardhanan, Discrete-time Sliding Mode Control: A Multirate Output Feedback Approach, Springer, Germany, 2006.

[19] K. Rajashekara, A. Kawamura, K. Matsuse, "Sensorless Control of AC Motor Drives, Speed and Position Sensorless Operation", IEEE Press, 1996.

[20] Y. Han, J. Choi, Y. Kim, "Sensorless PMSM drive with a sliding mode control based adaptive speed and stator resistance estimator", IEEE Transactions on Magnetics, Vol. 36, Issue 5, Part 1, Sept 2000 pp. 35883591.

[21] S. Alahakoon, K. Walgama, M. Leksell and L. Harnefors: "An Investigation into a Sensorless Adaptive Control Scheme Based on a Voltage Error Vector for Permanent-Magnet Synchronous Motors", Proceedings of the 33rd International Conference on Intelligent Motion, Nürnberg, Germany, May 1998, pp. 203-212.

[22] O. Wallmark, L. Harnefors, O. Carlson, "An improved speed and position estimator for salient permanent-magnet synchronous motors", IEEE Transactions on Industrial Electronics, Vol. 52, Issue 1, Feb. 2005 pp $255-262$.

[23] S. Alahakoon, "Digital Motion Control Techniques for Electrical Drives", Ph.D. Thesis, Electrical Machines and Power Electronics, Dept. of Electric Power Engineering, Royal Institute of Technology, Stockholm, Sweden, Oct. 1999, ISBN - 91 - 7170 - 555 - 4. 